\title{
Phase Separation on Bicontinuous Cubic Membranes: Symmetry Breaking, Reentrant, and Domain Faceting
}

\author{
Fabien Paillusson, ${ }^{1,2, *}$ Matthew R. Pennington, ${ }^{1}$ and Halim Kusumaatmaja ${ }^{1, \dagger}$ \\ ${ }^{1}$ Department of Physics, Durham University, South Road, Durham DH1 3LE, United Kingdom \\ ${ }^{2}$ School of Mathematics and Physics, University of Lincoln, Brayford Pool, Lincoln LN6 7TS, United Kingdom
}

(Received 27 August 2015; published 26 July 2016)

\begin{abstract}
We study the phase separation of binary lipid mixtures that form bicontinuous cubic phases. The competition between the nonuniform Gaussian membrane curvature and line tension leads to a very rich phase diagram, where we observe symmetry breaking of the membrane morphologies and reentrant phenomena due to the formation of bridges between segregated domains. Upon increasing the line tension contribution, we also find faceting of lipid domains that we explain using a simple argument based on the symmetry of the underlying surface and topology.
\end{abstract}

DOI: 10.1103/PhysRevLett.117.058101

Introduction.-Lipid self-assembly can adopt an astonishing range of shapes and morphologies, from single bilayer structures to stacks and convoluted periodic structures [1]. Nature has, of course, exploited this polymorphism. A large number of organelles feature lipid-based structures, including synaptic vesicles, the endoplasmic reticulum, and the Golgi apparatus. At the same time, lipids are indispensable for the detergency and foodstuffs industries [2], and membrane-based structures are increasingly exploited in biotechnological and biomedical applications, e.g., as efficient nanoporous scaffolds for tissue engineering [3] or for gene silencing with siRNA [4].

In this Letter we will focus on one particular type of mesophase that lipid mixtures in water can adopt, the so-called bicontinuous cubic phases (BCPs) [5-7], whereby the lipids form a triply periodic lipid bilayer that separates two percolating and nonintersecting water channels [8-10]. These phases have attracted attention due to their high surface area, continuity of the bilayer surface, and pore network. The amphiphilic nature of the lipids also allows other molecules to be embedded in them; for example, they have a high propensity to enable membrane protein crystallization. Although the details remain unclear, it is thought that a combination of curvature induced phase separation on the cubic surface, a local destabilization of the cubic phase to a lamellar phase, and a two-dimensional reservoir of proteins provided by the cubic phase are responsible for the observed yield [11]. Here, our interests are in the aforementioned curvature induced phase separation.

Both in the biological and synthetic systems, these lipid mesophases usually contain more than one lipid species.

Published by the American Physical Society under the terms of the Creative Commons Attribution 3.0 License. Further distribution of this work must maintain attribution to the author(s) and the published article's title, journal citation, and DOI.
To the best of our knowledge, the distribution of different lipids across such a cubic surface, especially the possible demixing transitions under the influence of the nonuniform curvature of the membrane structures, is still not well understood. Most studies on lipid phase separation focus on much simpler membrane geometries, such as lipid vesicles and supported membranes [12-14]. From a biological perspective, lateral lipid organizations into domains and membrane curvatures are ubiquitous features, and are known to play an important role for the membrane functionalities $[15,16]$. From a materials perspective, understanding the distribution of the species of interest on a BCP may be the first steps towards a systematic and rational functionalization of BCPs, where the active species can be localized into targeted domains. Finally, our work provides a comprehensive phase diagram, with predictions of the distinguishing features, which we hope will stimulate experimental verifications.

This Letter is organized as follows. We first show that if the two species do not interact but induce different bending rigidities, then a single type of curvature induced phase separation occurs at all nonzero area fractions. Upon considering the interactions between the species, we observe a multiplicity of new modalities for the phase separation, including the formation of bridges between previously disconnected lipid domains. Moreover, we observe the faceting of domains for which we provide a simple explanation relying on symmetry and topology.

Segregation in the absence of line tension.-In this Letter we consider a binary lipid mixture or, alternatively, a mixture of lipids and proteins that has formed a minimal surface $S$ (with zero mean curvature everywhere), and ask what the thermodynamically favored repartitions of the species are and how they depend on the bending rigidities and interspecies interactions. Here, our focus is on the triply periodic surfaces that are known to be formed by lipid mixtures as well as mixtures of lipids and proteins in water 
[5-7]. We use the standard notations $P, D$, and $G$ for primitive, diamond, and gyroid surfaces, respectively. Since they are periodic, we characterize their properties per unit cell. To model a binary mixture on a curved surface, we use a straightforward extension of the Helfrich Hamiltonian [17] to the case of a binary mixture on a minimal surface that reads [18-21]

$$
H_{\mathrm{el}}^{S}\left(f_{A}\right)=\delta \kappa \int_{S} d \mu_{S}(x) \sigma_{A}(x) G(x),
$$

where $x$ denotes a point on $S, d \mu_{S}(x)$ is the area measure on $S$ at $x, G(x)<0$ is the Gaussian curvature at point $x, f_{A}$ is the imposed area fraction of species $A$ (with $f_{B}=1-f_{A}$ ), the field $\sigma_{A}(x) \in[0,1]$ is the mean occupation number of species $A$ at $x, \delta \kappa=\kappa_{g}^{A}-\kappa_{g}^{B}$, and $\kappa_{g}^{A, B}$ are the Gaussian bending rigidities associated with the species $A$ and $B$, respectively. Our convention here is $\kappa_{g}^{A}<\kappa_{g}^{B}$, such that $\delta \kappa<0$. It is also worth remarking that, since typically $\kappa_{g}<0, B$ domains are softer than $A$ domains. Such a model may represent a coexistence between $L_{o}$ (liquid ordered, $A$ rich) and $L_{d}$ (liquid disordered, $B$ rich) domains, or alternatively, between lipid-rich and protein-rich domains.

To model the distribution $\sigma_{A}$ in a more tractable way, we first stress that any minimal surface with genus $g$ embedded in a flat torus $\mathbb{T}^{3}$ must contain $4(g-1)$ zero Gaussian curvature points [22]. For the $P, D$, and $G$ surfaces, $g=3$ and they have eight zeros. Each zero is located at the center of a hexagonal area we term a patch (See Fig. 1 here and Fig. 1 in Supplemental Material (SM) [18]). The unit cell of either of the $P, D$, or $G$ surfaces can thus be partitioned into eight equivalent patches $\left\{\Sigma_{i}\right\}_{i=1, \ldots, 8}$ such that the unit cell surface $S=\cup_{i=1}^{8} \Sigma_{i}$. We characterize the repartition of the lipids on $S$ by both the area fraction $f_{A}^{i}$ of lipid $A$ on each patch $\Sigma_{i}$ and the occupation number function $\sigma_{A}^{i}(x)$ in it. For each patch $\Sigma_{i}$, given $\sigma_{A}^{i}$, the entropy then reads $\mathbb{S}_{i}=-k_{B} \int_{\Sigma_{i}} d \mu_{S}(x)\left[\sigma_{A}^{i} \ln \sigma_{A}^{i}+\left(1-\sigma_{A}^{i}\right) \ln \left(1-\sigma_{A}^{i}\right)\right]$.

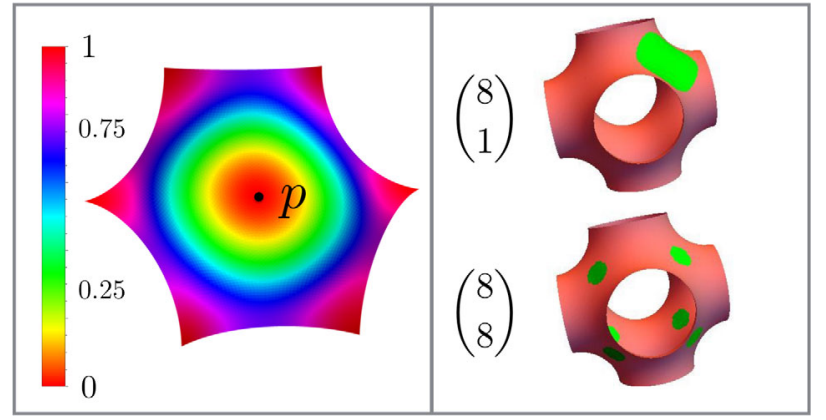

FIG. 1. Visualizing the $P$ surface. Left panel: normalized Gaussian curvature field $\left(G(x) / G_{\min }\right)$ [note that $G(x) \leq 0$ ] on a single patch with the zero-curvature point $p$ at its center. Right panel: curvature induced formation of $A$-lipid domains (in green) in $k$ patches among the eight available denoted by $\left(\begin{array}{l}8 \\ k\end{array}\right)$ (for $\left.f_{A}=0.07\right)$. For the sake of illustration we show them for $k=1$ (top) and $k=8$ (bottom).
Minimizing the overall free energy $\mathcal{F}=H_{\mathrm{el}}^{S}\left(f_{A}\right)-T \sum_{i=1}^{8} \mathbb{S}_{i}$ with respect to the occupation number for a given set of area fractions $\left\{f_{A}^{i}\right\}_{i=1, \ldots, 8}$ leads to the typical Fermi-Dirac distribution $\sigma_{A}^{i *}(x)=\left[1+e^{-\beta \lambda_{A}^{i}+\beta \delta \kappa G(x)}\right]^{-1}$, where $\lambda_{A}^{i}$ is a Lagrange multiplier that imposes the value of $f_{A}^{i}$.

At low temperatures, the Fermi-Dirac distribution will reach a value close to unity for all points $x$ of $\Sigma_{i}$ with an energy lower than $\lambda_{A}^{i}$. The lowest energy point $p_{i}$ in a patch $\Sigma_{i}$ is the symmetry point of the patch, which has an exactly zero Gaussian curvature (cf. Fig. 1). Thus, at low $T$, the lipids $A$ will fill the neighborhood of $p_{i}$ until they reach a critical Fermi curve $\mathcal{C}_{F}$, where $\left\{x \in \mathcal{C}_{F} \mid \delta \kappa G(x)=\lambda_{A}^{i}\right\}$, beyond which there are no more lipids of type $A$ (cf. Fig. 1; a disconnected area occupied by lipid $A$ is termed a domain). Close to $p_{i}$, one may use polar coordinates $\left(r_{i}, \theta_{i}\right)$ and, as a crude approximation, the space is assumed Euclidean and circularly symmetric near $p_{i}$. This allows us to Taylor expand the function $G$ about $p_{i}$ up to second order so that the curvature energy reads $H_{\mathrm{el}}^{\Sigma_{i}}\left(f_{A}^{i}\right) \sim \delta \kappa \int_{0}^{R_{i}} 2 \pi r_{i} d r_{i}\left[G^{\prime \prime}\left(p_{i}\right) r_{i}^{2} / 2\right] \sim C\left(f_{A}^{i}\right)^{2}$, where $R_{i}$ is the mean radial distance of the Fermi curve from the point $p_{i}$ such that $f_{A}^{i} \approx \pi R_{i}^{2} / \mu\left(\Sigma_{i}\right)$. Here, $C$ is a constant and $\mu\left(\Sigma_{i}\right)$ is the area of the hexagonal patch $\Sigma_{i}$. Remarkably, in spite of the very crude approximations we have used, the predicted behavior of the curvature energy $H_{\mathrm{el}}^{\Sigma_{i}}\left(f_{A}^{i}\right) \propto\left(f_{A}^{i}\right)^{\alpha}$, with $\alpha=2$, is close to what we observed in simulations for the $P$ surface where the exponent is found to be $\alpha=1.83$ [18].

Next, upon minimizing the total free energy $\mathcal{F}=$ $C \sum_{i=1}^{8}\left(f_{A}^{i}\right)^{2}$ with respect to the area fractions $f_{A}^{i}$ at fixed total area fraction $f_{A}=\left(\sum_{i=1}^{8} f_{A}^{i}\right) / 8$, it is easy to see that the ground state in the repartition among the patches is always $f_{A}^{i}=f_{A}$ for all values of $f_{A}$, corresponding to the $\left(\begin{array}{l}8 \\ 8\end{array}\right)$ configuration in Fig. 1.

Effect of the line tension.-We have seen that, with only curvature, the $A$ lipids are evenly distributed among the eight available patches and form dense domains in the neighborhood of the zero curvature points at low temperature. We now consider how this picture changes if the $A-B$ interactions are not negligible, i.e., if there are line tension effects arising with domain formation, which is a more realistic physical scenario. To answer this question, we now carry out computer simulations of the binary phase separation on the $P$ surface (the qualitative picture is the same for the $D$ and $G$ surfaces, as justified in [18]). There are several known approaches to model bicontinuous cubic membranes, from coarse-grained molecular dynamics simulations [23] to continuum field theoretical approaches [24-26]. Here, we use Metropolis Monte Carlo simulations [27] to resolve the thermodynamics of the system.

In our approach, we explicitly discretize a piece of the $P$ surface contained in a cubic cell. This can be efficiently done with the help of the Weierstrass-Enneper representation of minimal surfaces $[22,28,29]$. Upon discretization, 
the binary mixture can then be modeled as an Ising-like problem (see SM [18] for technical details). A spin variable $s$ is associated with each site and takes either the value 0 (for species $B$ ) or 1 (for species $A$ ). The curvature Hamiltonian of Eq. (1) thus maps exactly onto a system of magnetic spins on a network $\mathcal{N}(S)$ with a nodedependent external magnetic field and reads

$$
H_{\mathrm{el}}^{S}\left(f_{A}\right)=\sum_{i \in \mathcal{N}(S)} \delta A_{i} \delta \kappa G_{i} s_{i}
$$

where $\delta A_{i}$ is the area of the tile $i$ on the surface. In this language, at any finite $f_{A}$, species $A$ (spin variable $s=1$ ) will occupy sites with the lowest value of $\delta A_{i} \delta \kappa G_{i}$ to minimize the total energy, as we have analyzed with a different vocabulary in the previous section. To model the $A-B$ interspecies interactions, we choose a short-range nearest neighbor interaction, which directly translates into the line tension of the lipid domains:

$$
H_{A-B}^{S}\left(f_{A}\right) \equiv J \sum_{i \in \mathcal{N}(S)} \sum_{j \in\langle i\rangle}\left(s_{i}+s_{j}-2 s_{i} s_{j}\right) \delta L_{i j},
$$

where $J$ sets the magnitude of the exchange interactions (line tension between the two species), $\delta L_{i j}$ is the length of the edge shared by cells $i$ and $j$, and $\left(s_{i}+s_{j}-2 s_{i} s_{j}\right)=1$ when $s_{i} \neq s_{j}$ and 0 otherwise.

Symmetry breaking. - As it is evident, the Hamiltonian in Eq. (3) is equivalent to an Ising model of ferromagnetism and therefore should lead to the same phenomenology: above a critical temperature $T^{*}(J)$, the system is paramagnetic and the two lipid species are mixed, while below $T^{*}$, the system becomes ferromagnetic and a symmetry breaking favoring the "lumping" of spins in spatial regions (segregation) occurs. There is, however, one crucial difference between the standard Ising model and our model. For the former, line tension effects always dominate demixing: domains of $A$ lipids coalesce to minimize the overall interfacial energy. In our model, this coalescence mechanism competes with the curvature-induced mechanism described in the previous section.

The first effect of line tension is to reorder the (energy) ranking of configurations $\left(\begin{array}{l}8 \\ k\end{array}\right)$ with the $k$ patches occupied by the $A$ species by shifting down the low $k$ configurations (because they have a lower interfacial cost) and up the high $k$ ones (because they have a high interfacial cost). A first account of the competition between the curvature and line tension consists in assuming that the total energy of a configuration $\left(\begin{array}{l}8 \\ k\end{array}\right)$ at a given packing fraction $f_{A}$ would read $k\left[H_{\mathrm{el}}^{\Sigma}\left(8 f_{A} / k\right)+H_{A-B}^{\Sigma}\left(8 f_{A} / k\right)\right]$, i.e., as the sum of the free energy of individual patches of equal size. This summation approximation is valid when isolated domains are formed at the center of the hexagonal patches, and one finds that increasing $f_{A}$ at fixed $J \ell /|\delta \kappa|$ always favors, eventually, higher $k$ values, in agreement with Monte Carlo simulation results shown in the phase diagram in Fig. 2(a).

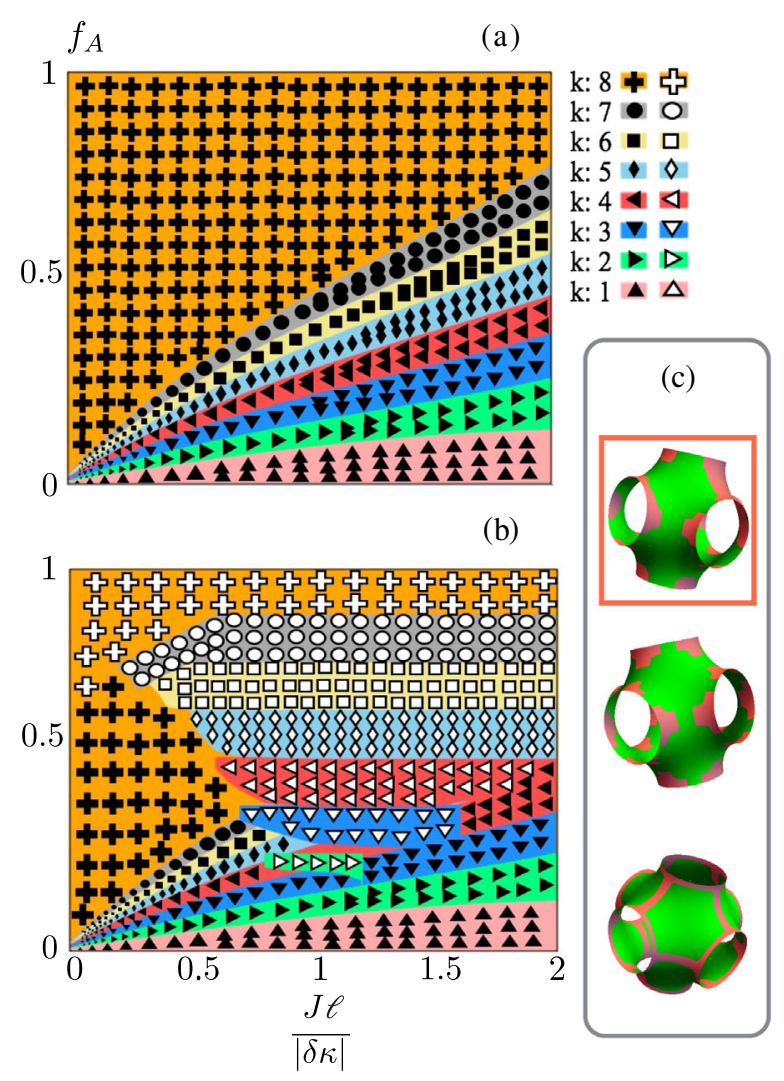

FIG. 2. Phase diagrams of the number of patches $k$ occupied by lipid $A$ domains among the eight available as a function of the area fraction $f_{A}$ and the ratio $J \ell /|\delta \kappa| . \ell$ is the lattice spacing of the cubic unit cell. For clarity, we show the phase diagrams (a) excluding (filled symbols) and (b) including (open symbols) the possibilities of bridge formations between lipid $A$ domains. In (c) are shown configurations with increasing energy from top to bottom for $f_{A}=0.75$ and $J \ell /|\delta \kappa|=0.5$. The boxed configuration with seven connected domains corresponds to the most stable phase.

The caveat is that this summation approach is only valid when the $A$ species domains are disconnected. Above certain $f_{A}$ values, the lowest energy configurations are in fact those in which domains of lipid $A$ span across multiple patches (see Fig. 2). These bridges between the patches essentially make the domains interact negatively and in a nonpairwise fashion. The location of these bridges coincides with the lowest curvature energy regions at the patch boundary (cf. Fig. 1). Taking these configurations into account, the phase diagram in Fig. 2(b) shows that the simple picture of Fig. 2(a) only holds for small $J \ell /|\delta \kappa|$ and $f_{A}$. In fact, one observes reentrant behaviors whereby a configuration $\left(\begin{array}{l}8 \\ k\end{array}\right)$ previously unfavored in the disconnected regime becomes refavored thermodynamically. We note that when bridges are formed in the $\left(\begin{array}{l}8 \\ 8\end{array}\right)$ configuration [open crosses in Fig. 2(b)], the segregated and continuous phases are effectively inverted (lipid $B$ domains are surrounded by $A$ ).

Domain faceting.-Another distinguishing feature that appears with line tension is the faceting of the domains 


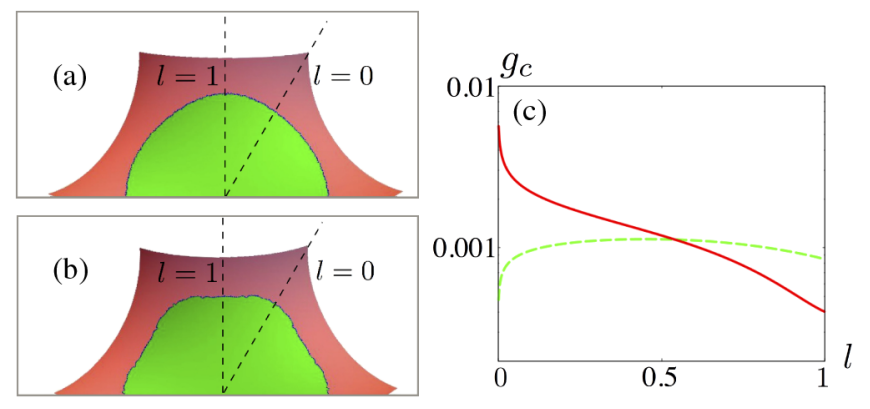

FIG. 3. Domain faceting. Shape of a lipid $A$ domain in the neighborhood of the zero-curvature point $p$ of a single hexagonal patch: (a) in the absence of line tension and (b) with high line tension $(J \ell /|\delta \kappa|>1)$. Note that only half of a patch is represented. (c) Geodesic curvature as a function of the curvilinear coordinate $l$ in the absence of line tension (dashed) and with high line tension (solid).

formed by the $A$ lipids. This effect is shown in Fig. 3(b) where the domain almost draws a hexagon compared to Fig. 3(a) where the shape is more rounded, thus the term "faceting." To explain this, we recall that in general if the underlying manifold has an $n$-fold rotational symmetry, we expect the bounding curve that minimizes the perimeter length of a domain with fixed area to be a regular $n$-gon whose sides are geodesics of the underlying manifold. Moreover, on an anisotropic curved surface, not all orientations of a regular $n$-gon are equivalent as they lead in principle to different total perimeter lengths. Thus, we interpret the bounding curve in Fig. 3(b) with sixfold symmetry to be the curve that minimizes both the shape and orientation at the same time.

To test the above rationale, we estimate the geodesic curvature along the bounding curves of the two representative examples shown in Figs. 3(a) and 3(b) (cf., e.g., Ref. [30]). In these figures, the curvilinear coordinate $l \in[0,1]$ is the normalized arc length of each curve, which enables the comparison of the geodesic curvature for curves with different total lengths in Fig. 3(c). In the absence of line tension, the geodesic curvature $g_{c}$ is approximately constant around the boundary. With a large line tension, $g_{c}$ reaches very high values for $l$ close to zero, but is much smaller than that without line tension as $l$ approaches 1 . This is consistent with the above explanation although it shows that the faceting is not perfect. It nevertheless sheds light on what happens as we approach the ideal faceting case: the geodesic vanishes almost everywhere except close to $l \simeq 0$ where it diverges. This divergence is representative of the wedge formed by the intersection of two geodesics of the 6-gon and whose angle $\gamma$ can be estimated to be $\gamma=2 \pi / 3-(6|\delta \kappa|)^{-1} H_{\mathrm{el}}^{\Sigma_{i}}\left(f_{A}^{i}\right)$ for an area $f_{A}^{i} \mu\left(\Sigma_{i}\right)[18]$.

Discussions.-Let us start by estimating where typical lipid mixtures are located in the phase diagram of Fig. 2. For a mixture of dioleoylphosphatidylcholine (DOPC), sphingomyelin, and cholesterol forming coexisting $L_{o}$ and $L_{d}$ domains, $J \simeq 1.2 \mathrm{pN}$ and $|\delta \kappa| \simeq 3 \times 10^{-19} \mathrm{~J} \quad[31,32]$.
Most synthetic BCPs, however, are formed using the lipid monoolein, which is known to have a low bending rigidity with $\left|\kappa_{G}\right| \sim \kappa_{m}<10 k_{B} T$ [33]. Here, $\kappa_{m}$ is the (mean curvature) bending rigidity. Using these values and taking the typical lattice spacing of a BCP $\ell \simeq 10-100 \mathrm{~nm}$ [34-36], this leads to $J \ell /|\delta \kappa|$ in the range of $O(0.1)-O(1)$ considered in this Letter.

When the line tension effects can be neglected, (a) the curvature of the surface alone is enough to induce segregation in all surfaces, and (b) the segregation is such that domains form in the same proportions on all available patches on the surface. We then confirmed this theoretical prediction by numerical calculations on the $P$ surface and looked at the effects of a nonzero line tension. The latter gives rise to two important features. (i) Below the demixing critical temperature, it favors the formation of bigger domains in a fewer number of patches available on the surface that we characterize with a corresponding phase diagram. We also observe reentrances in this patch-occupation space due to the formation of bridges between domains on neighboring patches. Some of these morphologies should lead to distinguishing features (e.g., different $x$-ray scattering signatures due to the change in symmetry), and we hope this work will stimulate experimental works to verify our predictions. (ii) In the large line tension limit, we observed a faceting of the domains for which we provided a simple explanation and that we can relate to the curvature energy of a domain on a patch.

Predicting patterning on cubic membranes is the vital first step towards their systematic and rational functionalization. On one hand, the ability to localize molecular species by design into targeted domains can be beneficial for controlled release in drug delivery or of chemical substances [37,38], and for templating self-assembly [39] or phase separation $[40,41]$ in the surrounding fluids. On the other hand, suppressing phase separation between lipid species or between lipids and proteins can be desirable in applications such as protein crystallization [11], where segregation at an incorrect stage can strongly hamper the efficiency of the applications.

There are also a number of avenues for future work. First, here we have assumed that the BCP remains a minimal surface. A closer inspection based on the theory developed in Ref. [42] for domain-induced budding shows that the conclusions presented here can be qualitatively affected when $\kappa_{m} /|\delta \kappa|<0.4$ [18]. However, estimates of this ratio for a wide range of lipid bilayers and monolayers in the literature show that it is only rarely below 1 [43]. This suggests that the minimal surface assumption is very reasonable for realistic parameter values. Further work is however still needed to fully assess how membrane deformation, including budding instability, affects the phase diagram of multicomponent BCPs. Second, the present work tacitly assumes that the membrane domains are formed by lipids of the same species in the two leaflets (registration phase). Indeed, recent work on flat bilayers 
suggests that registered domains is the thermodynamically favored phase for a wide range of lipid mixtures [44]. It would be interesting to relax this assumption to probe how curvature affects registration or antiregistration and how, in turn, registration or antiregisteration may affect the bilayer morphology. Third, the system considered here provides an excellent setup to study how nonuniform curvature may affect the nature of the demixing phase transition.

Research data supporting this Letter can be accessed at Ref. [45].

We thank J. M. Seddon, P. D. Olmsted, J. J. Williamson, N. J. Brooks, D. Frenkel, F. Schmid, and C. Semprebon for useful discussions, and the Biophysical Sciences Institute in Durham for support though a summer studentship for M. R. P. This work is funded by EPSRC (Grant No. EP/ J017566/1).

*Corresponding author

fpaillusson@lincoln.ac.uk

${ }^{\dagger}$ Corresponding author.

halim.kusumaatmaja@durham.ac.uk

[1] J. M. Seddon and R. H. Templer, in Structure and dynamics of membranes: From cells to vesicles, Handbook of Biological Physics, Vol. 1, edited by R. Lipowsky and E. Sackmann (Elsevier Science Publishers B.V., New York, 1995), Chap. 3.

[2] L. Sagalowicz and M. E. Leser, Curr. Opin. Colloid Interface Sci. 15, 61 (2010).

[3] S. C. Kapfer, S. T. Hyde, K. Mecke, C. H. Arns, and G. E. Schröder-Turk, Biomaterials 32, 6875 (2011).

[4] C. Leal, N. F. Bouxsein, K. K. Ewert, and C. R. Safinya, J. Am. Chem. Soc. 132, 16841 (2010).

[5] R. H. Templer, J. M. Seddon, N. A. Warrender, A. Syrykh, Z. Huang, R. Winter, and J. Erbes, J. Phys. Chem. B 102, 7251 (1998).

[6] B. Angelov, A. Angelova, M. Ollivon, C. Bourgaux, and A. Campitelli, J. Am. Chem. Soc. 125, 7188 (2003).

[7] A. I. I. Tyler, H. M. G. Barriga, E. S. Parsons, N. L. C. McCarthy, O. Ces, R. V. Law, J. M. Seddon, and N. J. Brooks, Soft Matter 11, 3279 (2015).

[8] A. D. Benedicto and D. F. O'Brien, Macromolecules 30, 3395 (1997).

[9] S. T. Hyde and G. E. Schröder-Turk, Interface Focus 2, 529 (2012).

[10] A. H. Schoen, Interface Focus 2, 638 (2012).

[11] M. Caffrey, Acta Crystallogr. F Struct. Biol. Commun. 71, 3 (2015).

[12] S. L. Veatch and S. L. Keller, Biophys. J. 85, 3074 (2003).

[13] R. Parthasarathy, C.-H. Yu, and J. T. Groves, Langmuir 22, 5095 (2006).

[14] T. Baumgart, A. T. Hammond, P. Sengupta, S. T. Hess, D. A. Holowka, B. A. Baird, and W. W. Webb, Proc. Natl. Acad. Sci. USA 104, 3165 (2007).

[15] D. A. Brown and E. London, Annu. Rev. Cell Dev. Biol. 14, 111 (1998).

[16] H. T. McMahon and J. L. Gallop, Nature (London) 438, 590 (2005).

[17] R. Lipowsky, Nature (London) 349, 475 (1991).
[18] See Supplemental Material at http://link.aps.org/ supplemental/10.1103/PhysRevLett.117.058101 for a derivation of the curvature Hamiltonian used in the manuscript and a survey of the Weierstrass representation of minimal surfaces and the Gauss-Bonnet theorem with their consequences for the phase separation problem. It also contains simulation and visualisation details of the study and a discussion of domain formation induced membrane bending/budding. The supplementary information includes additional Refs. [19-21].

[19] K. A. Brakke, Exp. Math. 1, 141 (1992).

[20] Wolfram Research, Inc., Mathematica (Wolfram Research Inc., Champaign, Illinois, 2016).

[21] N. Metropolis, A. Rosenbluth, M. Rosenbluth, A. Teller, and E. Teller, J. Chem. Phys. 21, 1087 (1953).

[22] W. H. Meeks, Indiana University Mathematics Journal 39, 877 (1990).

[23] M. Fuhrmans, V. Knecht, and S. J. Marrink, J. Am. Chem. Soc. 131, 9166 (2009).

[24] U. S. Schwarz and G. Gompper, Phys. Rev. E 59, 5528 (1999).

[25] W. B. Lee, R. Mezzenga, and G. H. Fredrickson, Phys. Rev. Lett. 99, 187801 (2007).

[26] S.-D. Yang, H. G. Lee, and J. Kim, Comput. Phys. Commun. 181, 1037 (2010).

[27] D. Frenkel and B. Smit, Understanding Molecular Simulation: From Algorithms to Applications (Academic Press, New York, 2001).

[28] U. Dierkes, S. Hildebrandt, A. Küser, and O. Vohlrab, Minimal Surfaces I: Boundary Value Problems (Springer, New York, 2013).

[29] P. Gandy and J. Klinowski, Chem. Phys. Lett. 322, 579 (2000).

[30] R. D. Kamien, Rev. Mod. Phys. 74, 953 (2002).

[31] S. Semrau, T. Idema, L. Holtzer, T. Schmidt, and C. Storm, Phys. Rev. Lett. 100, 088101 (2008).

[32] T. Baumgart, S. T. Hess, and W. W. Webb, Nature (London) 425, 821 (2003).

[33] G. C. Shearman, O. Ces, R. H. Templer, and J. M. Seddon, J. Phys. Condens. Matter 18, S1105 (2006).

[34] H. M. G. Barriga, A. I. I. Tyler, N. L. C. McCarthy, E. S. Parsons, O. Ces, R. V. Law, J. M. Seddon, and N. J. Brooks, Soft Matter 11, 600 (2015).

[35] Z. A. Almsherqi, S. D. Kohlwein, and Y. Deng, J. Cell Biol. 173, 839 (2006).

[36] Y. Deng, M. Marko, K. F. Buttle, A. Leith, M. Mieczkowski, and C. A. Mannella, J. Struct. Biol. 127, 231 (1999).

[37] S. B. Rizwan, B. J. Boyd, T. Rades, and S. Hook, Expert Opin. Drug Deliv. 7, 1133 (2010).

[38] N. Garti, D. Libster, and A. Aserin, Food Funct. 3, 700 (2012).

[39] L.-L. Pontani, M. F. Haase, I. Raczkowska, and J. Brujic, Soft Matter 9, 7150 (2013).

[40] M. Böltau, S. Walheim, J. Mlynek, G. Krausch, and U. Steiner, Nature (London) 391, 877 (1998).

[41] L. Xue, J.Zhang, and Y. Han, Prog. Polym. Sci. 37, 564 (2012).

[42] R. Lipowsky, J. Phys. II (France) 2, 1825 (1992).

[43] M. Hu, J. J. Briguglio, and M. Deserno, Biophys. J. 102, 1403 (2012).

[44] J. J. Williamson and P. D. Olmsted, Biophys. J. 108, 1963 (2015).

[45] DOI: 10.15128/r2vd66vz88d. 